\section{Molecular Authorities}

When you need information on a human protein, who are you gonna call? Dr. Akhilesh Pandey and his team of biologists, bioinformaticists, and software engineers at Johns Hopkins University hope you'll dial up their interesting Web project, the Human Protein Reference Database (HPRD). The site's collection of over 3300 proteins, 13,000 protein-protein interactions, and 25,000 references is a treasure trove of scientific data. More impressive, though, is the database's design-put together using open source software and thousands of hours of effort by human (not machine) curators. Access is free for noncommercial usage, and fees generated by scientists with commercial application will be used solely to maintain the system. Sound interesting? Then contribute to the cause by filling out a form to be a "molecule authority" and help ensure the accuracy of data on one or more proteins in the database.

\section{www.hprd.org}

\section{Genome Ticker}

Quick, how many genome projects are in progress, which ones have been published, and what's the tally of prokaryotes, archaeans, and eukaryotes? Unsure? You're not alone. Genomic sequencing efforts seem to grow in scope and size weekly. Fortunately, someone's keeping track. Genomesonline, or GOLD, as it is better known, reported an early November tally of a whopping 941 genome projects completed or in the pipeline. GOLD's database is searchable, with fields for genome size, organism name, hyperlinks to the sequence file(s), organization performing the work, and reference info, as appropriate. Access to the information is simple, easy, and best of all, free, for academic or nonprofit use.

\section{www.genomesonline.org}

\section{When Good Genes Go Bad}

Most of the time, the 30,000+ genes in the human genome behave like a well-trained army, marching from parents to progeny in tight, synchronized steps, but occasionally a gene goes AWOL, a bad set of alleles pair, or an error occurs upon fertilization. When genetic mayhem ensues, researchers and physicians alike can turn to the outstanding GeneTests page on the geneclinics.org site for informative articles and links to over 500 laboratories that perform genetic testing. Funded by the National Institutes of Health $(\mathrm{NIH})$, the Health Resources and Services Administration (HRSA), and the

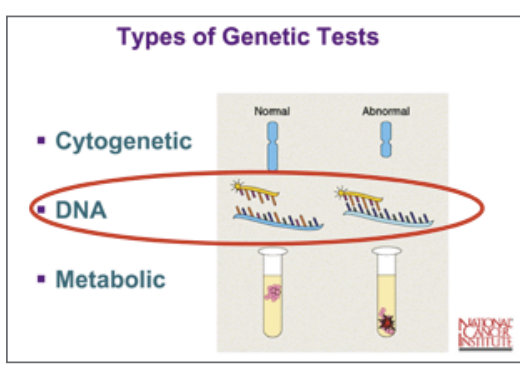

U.S. Department of Energy (DOE), GeneTests contains 220+ expert-authored articles called GeneReviews that summarize clinical aspects of each disease and the likelihood of its occurrence in siblings. With reports that are hyperlinked and well-written, useful information and understanding is only a click away.

\section{www.geneclinics.org}

Image from GeneTests: Medical Genetics Information Resource (database online). Copyright, University of Washington and Children's Health System, Seattle. 1993-2003. Updated weekly. Available at http://www.genetests.org. Accessed 2 December 2003

\section{A Fly by Any Other Name}

A quick scan through the FlyNome database of Drosophila nomenclature reminds us that fruitfly researchers don't expend all of their creativity on technical matters. The fanciful names of mutant Drosophila genes, such as breathless (defective trachea development), fear of intimacy (gonadal mesoderm cells fail to coalesce tightly), and jelly belly (lack of gut muscles), not only bring a smile to the face, but they also help to paint a mental image of the defect. Researchers new to the field will embrace FlyNome for its help in making sense of a jargonfilled world. Records include the names and stories of how the genes came to be named, as well as the relevant journal references.

\section{www.flynome.com}

\section{Cellular Hub}

The products of the cellular myc genes are members of one of the cell's best connected families. They can stoke metabolic fires, affect progression through the cell cycle, and when malfunctioning, lead to neoplasia. Making sense of the myriad of actions of myc is the business of the Myc Cancer Gene site,

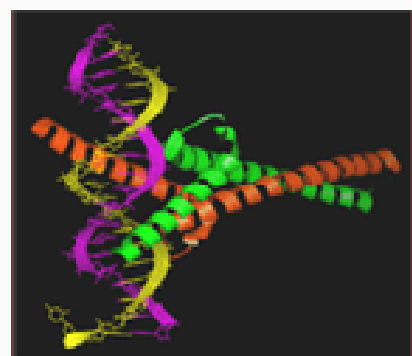

whose databases track (i) genes affected by myc's transcription regulating functions (1382 and counting at press time), and (ii) myc's role in human cancers. Transcription database entries can be sorted or, more conveniently, accessed by a search engine. Returned records contain information in up to 21 fixed fields and include hyperlinks to the National Center for Biotechnology Information (NCBI) Unigene entries for easy access to additional information.

\section{wWw.myccancergene.org}

\section{by Kevin Ahern}

Please send Web site recommendations to ahernk@orst.edu 